\section{IMPLEMENTACIÓN DE LA MODALIDAD B-LEARNING EN LA ASIGNATURA OPTATIVA ERGONOMÍA VISUAL}

\author{
Francisco Miguel Martínez Verdú \\ Departamento de Óptica, Farmacologia y Anatomia, \\ Universidad de Alicante \\ Carretera de San Vicente del Raspeig s/n, 03690 Alicante \\ verdu@ua.es \\ http://blogs.ua.es/verduset70/category/ergonomia-visual/
}

\section{IMPLEMENTATION OF THE B-LEARNING METHOD IN THE ELECTIVE SUBJECT: "ERGONOMÍA VISUAL" VISUAL ERGONOMICS}

\begin{abstract}
Along past second semester of course 2007-08, at first time a new optional subject, Visual Ergonomics, was taught by blearning methodology in Graduate of Optics and Optometry at the University of Alicante. In this word the some issues most outstanding , both positive and negative, are described in this first implementation with a reduced group of students. The theoretical section was mainly developed by $b$-learning methodology interchanging on-class training, the most of them as feed-back, with virtual sessions supported by some web 2.0 digital tools as a blog, multimedia and interactive sessions, and team work (where teacher participates as one additional member, inside a strategy pre-conceived of situational leadership applied to classroom). The informal opinion polls, collected in course final, show that, in spite of the fact the students were conscious they had clearly approved this subject, the vast of them did not friendly accept so educational innovations in this short period. Then, as epilogue to this experience in education, this subject could not teach in course 2008-09 because any student is enrolled. Although this discipline is highly recommended from national guidelines for future Graduate in Optics and Optometry, it is not sure it continues at the University of Alicante. However, I hope the positive issues of this experience in education can be extrapolated with success in other pre and university degrees.
\end{abstract}

KEY WORDS: Experiences in education; general skills and their development; research in education; pedagogical and didactical innovations; technological issues; internet based technologies; educational software; computer supported collaborative work; knowledge management; situational leadership.

\section{INTRODUCCIÓN}

La ergonomía visual permite tomar y aplicar de forma sinérgica los conocimientos y habilidades adquiridos sobre Óptica y Optometría durante toda la carrera de grado (de momento,
RESUMEN: Durante el segundo cuatrimestre del curso pasado (2007-08) se implementó por primera vez la modalidad b-learning en la asignatura optativa Ergonomía Visual de la Diplomatura de Óptica y Optometría de la Universidad de Alicante. En este trabajo se describen los aspectos más relevantes, tanto positivos y negativos, de esta primera implementación con un grupo reducido de alumnos. La parte teórica se ha desarrollado principalmente en modalidad blearning alternando sesiones presenciales, la mayoria de ellas como retroalimentación, con sesiones virtuales apoyadas mediante varias herramientas digitales web 2.0 como un blog, sesiones multimedia e interactivas, y trabajo en equipo (donde el profesor participa como uno más, dentro de una estrategia preconcebida de liderazgo situacional aplicado al aula). Las encuestas informales recogidas a final de curso muestran que, a pesar de que los alumnos eran conscientes de que todos habian aprobado con creces la asignatura, la mayoría de ellos no aceptaron de buen grado tantas innovaciones educativas en tan poco tiempo. Así pues, y como epílogo a esta experiencia educativa, esta asignatura no se puede impartir en el curso 2008-09 porque ningún alumno se ha matriculado. Siga adelante o no esta asignatura en el futuro grado, aun siendo una materia recomendada en las directrices ministeriales de esta carrera universitaria, espero que los aspectos positivos de esta experiencia educativa se puedan extrapolar con mayor éxito en otros estudios universitarios y preuniversitarios.

PALABRAS CLAVE: Experiencias educativas; habilidades generales y su desarrollo; investigación en docencia; innovaciones pedagógicas y didácticas; aspectos tecnológicos; tecnologías basadas en Internet (blog, etc.); software educacional; trabajo colaborativo por ordenador; gestión de conocimiento; liderazgo situacional.

diplomatura de tres cursos académicos) para identificar y analizar los factores de riesgo medioambientales y laborales que pueden causar problemas de salud ocular o de funcionalidad visual. La asignatura sirve para fomentar al estudiante en la aplicación de los conocimientos asimilados en la reso- 
lución de problemas prácticos relacionados con el estudio de la ergonomía visual. También fomentará la aplicación de los pasos básicos para prescribir correctamente un tratamiento para restaurar la función visual o mejorar el rendimiento visual. Es, por tanto, una asignatura con marcado carácter interdisciplinar (que necesita la cooperación de otras disciplinas) y multidisciplinar (que abarca o afecta a varias disciplinas), ya que al basarse en conocimientos amplios de Óptica y Ciencias de la Visión redunda de nuevo en estas materias y otras afines. Por tanto, al estar la asignatura centrada en la interacción ojo-entorno-tarea (herramienta o máquina), ésta sirve de trampolín para el posterior desarrollo de proyectos teóricos y aplicados donde la visión humana juegue un papel importante en el desarrollo y la optimización de procesos industriales, y, en la seguridad y confort visual de actividades laborales y de ocio.

Esta materia, como asignatura optativa en la Diplomatura de Óptica y Optometria de tercer curso, tiene una carga lectiva de seis créditos (equivalentes a 7,5 ECTS), 4,5 teóricos y 1,5 prácticos, y con una matrícula anual media de 15 alumnos (para el curso 2007-08 fueron 13 alumnos). Actualmente, en el curso 2007-08 esta asignatura está enmarcada como experiencia piloto dentro de una convocatoria de innovación educativa del Vicerrectorado de Tecnología e Innovación Educativa de la Universidad de Alicante. En particular, para este curso académico, y los siguientes, la asignatura se va impartir en modalidad docente b-learning (aprendizaje semipresencial), de forma que 15 horas de los créditos teóricos se van a impartir en esta modalidad de aprendizaje. Esto implica cambiar el paradigma formativo, no basado solamente en un paradigma docente (apoyado en la clase magistral), sino aplicar también el paradigma basado en el aprendizaje (orientado en la participación más activa del estudiante, tanto a nivel individual como en grupo). Para ello, la clave estará en mantener las fortalezas de la clase magistral, pero proponer también actividades reales y sobre todo virtuales que minimicen sus debilidades, tal como se sugiere en el blog innovacioneducativa.wordpress.com, en la entrada "Reducir las clases teóricas un diagnóstico unánime, pero... ¿cómo reducirlas?". Por tanto, el objetivo formativo principal es demostrar al estudiante que puede aprender bastante disfrutando a través de numerosas actividades individuales y grupales sin que el profesor reduzca el nivel de exigencia en la adquisición de conocimientos, habilidades y capacidades asociadas a la asignatura, y que la participación activa del estudiante en la asignatura puede suponer también un crecimiento positivo a nivel personal como de sus compañeros, pero también para el profesor y para la asignatura en cursos venideros.

\subsection{Objetivos didácticos y competencias adquiridas}

Los objetivos didácticos generales son:

OG1- Identificar y analizar los factores de riesgo medioambientales y laborales que pueden causar problemas de salud ocular o de funcionalidad visual;

0G2- Fomentar al estudiante para aplicar los conocimientos obtenidos en esta asignatura en la resolución de problemas prácticos relacionados con el estudio de la ergonomía visual;

0G3- Fomentar al estudiante para prescribir correctamente un tratamiento para restaurar la función visual o mejorar el rendimiento visual;

OG4- Proporcionar a los estudiantes los recursos bibliográficos adecuados para que ellos puedan educar al paciente en la prevención de riesgos oculares y visuales;

0G5- Estimular en el alumno, de forma individual, cooperativa y en trabajo en equipo, en el uso de las nuevas tecnologías de apoyo a la docencia con el fin de llegar a ser conscientes de que es posible aprender disfrutando y alcanzar un rendimiento académico notable.

Los objetivos cognitivos son:

0C1- Comprender la interconexión de las tres piedras angulares de la ergonomía (usuario-tarea-entorno) y su implementación en el campo de las Ciencias de la Visión.

OC2- Valorar los factores de riesgo ocular en actividades laborales y de ocio por exposición de energía radiante, ya sea asociada a lámparas de espectro continuo o a láseres, y comprender las bases del control radiométrico para evitar tales lesiones oculares.

0C3- Adquirir una panorámica general de todos los aspectos del diseño de iluminación y color (fotometría, selección de lámparas/luminarias, métodos de alumbrado, etc.) para multitud de ambientes (interiores y/o exteriores), y su relación con el rendimiento visual del usuario. 
0C4- Identificar y analizar los factores de riesgo medioambientales y laborales que pueden causar lesiones oculares de cualquier tipo (mecánicas, químicas, etc.).

0C5- Conocer los límites funcionales de la visión humana y su relación con la edad, ya sea en el ámbito laboral y en actividades de ocio, en conexión con los factores de visibilidad asociados a la tarea.

0C6- Adquirir una panorámica general de todos los aspectos implicados en la fatiga y rendimiento visual de actividades como: el uso de pantallas de visualización de datos, la conducción de vehículos, la práctica deportiva, y, en el aprendizaje.

Los objetivos procedimentales (destrezas a adquirir) son:

OP1- seleccionar un filtro óptico de protección frente a radiaciones ópticas, incluidas las procedentes de láseres;

OP2- diseñar un entorno luminoso y cromático, ya esté asociado a un ambiente de interior (oficina, etc.) o de exterior;

OP3- seleccionar un equipo de protección individual (EPI) ocular según los factores de riesgo en el entorno laboral;

OP4- elaborar el esquema general de un programa de prevención ocular en cualquier tipo de empresa;

OP5- conocer y localizar toda la normativa española, europea y mundial referente a la protección ocular;

OP6- elaborar un estándar visual laboral y elegir cuando mejor convenga un método de análisis industrial de visión o una práctica optométrica modificada;

OP7- establecer y aplicar una lista priorizada de recomendaciones, según cada caso, para disminuir la fatiga visual delante de pantallas de ordenador;

OP8- establecer y aplicar una lista priorizada de recomendaciones, según cada caso, para aumentar el rendimiento visual (y global) de las personas en tareas como la conducción, la práctica deportiva o en el ambiente educativo.

Y los objetivos actitudinales (habilidades sociales) son:

OA1- Trabajo en equipo: división de roles y tareas de un grupo de trabajo (prácticas, etc.).

OA2- Habilidades en las relaciones interpersonales: capacidad de atención en clase.
OA3- Razonamiento crítico: adquisición de iniciativa para preguntar al profesor, ya sea de forma presencial o virtual.

OA4- Aprendizaje autónomo y formación continuada: concienciación de las ventajas del aprendizaje autónomo, compartido, planificado y la formación continua.

OA5- Adaptación a nuevas situaciones: capacidad de adaptación a procedimientos experimentales con personas reales y utilización de nuevas tecnologías de apoyo a la docencia.

OA6- Iniciativa y espíritu emprendedor.

OA7- Motivación por la calidad.

Las competencias adquiridas (académicas, científicas y profesionales) por el estudiante en esta asignatura se desglosan a continuación:

- Cognitivas: análisis de tareas que pueden causar problemas oculares y visuales;

- Metodológicas: capacidad de análisis y síntesis, y, aprender a pensar y aplicar Ergonomía de la Visión;

- Destrezas tecnológicas: diseño de iluminación, entrenamiento visual, manejo de fuentes bibliográficas, herramientas de web 2.0;

- Destrezas lingüísticas: jerga técnica, preparación de exposición en público, comunicación oral en público

\subsection{Temario}

Bloque I. Introducción a la Ergonomía Visual Tema 1: Introducción a la Optometría Ocupacional

Bloque II. Factores ambientales

Tema 2: Efectos oculares de la energía radiante

Bloque III. El entorno de iluminación y color

Tema 3: Luminotecnia

Tema 4: El entorno cromático

Bloque IV. Protección ocular en el trabajo Tema 5: Protección ocular en el trabajo

Bloque V. Rendimiento visual en el trabajo Tema 6: Capacidad visual

Tema 7: Pantallas de visualización de datos Tema 8: Visión en la conducción

ARBOR CLXXXV EXTRA 2009 125-138 ISSN: 0210-1963

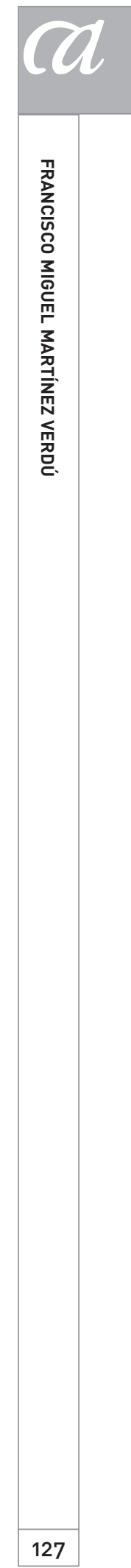

doi: 10.3989/arbor.2009.extran1210 
Tema 9: Visión y deporte

Tema 10: Visión y educación

Y las sesiones prácticas de laboratorio son:

Práctica n. ${ }^{0}$ : Fotometría de fuentes extensas

Práctica n. ${ }^{2}$ : Caracterización de luminarias

Práctica n. ${ }^{\circ}$ : Diseño de iluminación: alumbrado interior

Práctica n. ${ }^{\circ}$ 4: Diseño de iluminación: alumbrado exterior

Práctica $n .{ }^{\circ}$ 5: Entrenamiento visual (I): memoria visual, taquitoscopio, sacádicos y seguimiento.

Práctica n. ${ }^{\circ}$ 6: Entrenamiento visual (II): percepción de forma, reconocimiento de patrones, hiperagudeza y conciencia periférica

Respecto a los prerrequisitos de la asignatura, ya que se trata de una asignatura optativa de tercer curso y de último cuatrimestre, los conocimientos teóricos y prácticos adquiridos previamente sobre Óptica y Visión (radiometría y fotometría, neurofisiología y percepción visual, optometría) se consideran más que suficientes. Por otra, se consideran importantes nociones generales sobre ergonomía y sobre las tecnologías actuales asociadas a actividades laborales y/o de ocio. Como plan de trabajo y actividades para la nivelación de estos prerrequisitos se han utilizado las sesiones asociadas al tema 1.

\subsection{Metodología docente y evaluación}

La metodología propuesta para la parte no presencial consiste básicamente en apoyarse en herramientas de web 2.0, consistentes principalmente en:

- Un blog de la asignatura, enmarcado dentro del blog personal del profesor/coordinador: http://blogs.ua.es/ verduset70/category/ergonomia-visual/.
- Uso de sesiones multimedia e interactivas en la plataforma digital de gestión de contenidos de aprendizaje de la UA (http://www.ua.es/es/univirtual/index. html), conocida habitualmente como Campus Virtual, mediante el uso del plug-in para MSWord denominado CourseGenie (ahora Wimba Create) para la generación del material del curso (temas, cuestiones de retroalimentación, inserción de enlaces web, animaciones, vídeos, etc.).

- Uso de Google Docs (http://docs.google.com/) como herramienta de trabajo en equipo, donde el profesor como un miembro más (dentro de una estrategia preconcebida de liderazgo situacional aplicado al aula, http://blogs.ua.es/verduset70/ 2008/04/23/liderazgosituacional-aplicado-al-aula-y-aprendizaje-informal/), participará en cada subgrupo para la elaboración de temas complementarios de exposición oral en clase a final de curso.

Todo esto servirá para desarrollar y evaluar trabajos individuales dirigidos (buscar recursos por Internet, caza del tesoro tipo test, trabajo o debate, etc.), trabajos cooperativos (puesta en común de trabajos individuales vía blog), y trabajo en equipo (vía Google Docs) en consonancia con los objetivos de aprendizaje marcados anteriormente, incluso los desglosados en objetivos cognitivos, procedimentales y actitudinales. Esto, a su vez, implica un cambio en el diseño y la organización de los contenidos de aprendizaje, $y$, aplicar feed-back sobre el usuario (alumno) y una acción tutorial continua (muchas veces proactiva, y no solamente reactiva), siempre a disposición del alumno.

El volumen estimado total de trabajo del alumno (VTTA, en horas) se desglosa a continuación:

TABLA 1: DESGLOSE DE ACTIVIDADES RELACIONADAS CON EL VOLUMEN DE TRABAJO TOTAL DEL ALUMNO

\begin{tabular}{|c|c|c|c|c|c|c|c|c|}
\hline ACT & PTi & PTCo & ACP & PTCP & EPE & RE & AT & \begin{tabular}{c} 
VTTA $=$ \\
$=187,5$ \\
\hline 30
\end{tabular} \\
\hline
\end{tabular}

ACT: asistencia a clase teórica.

PTi: preparación de trabajos individuales.

PTCo: preparación de trabajos cooperativos.

ACP: asistencias a clase práctica (laboratorio).
PTCP: preparación de trabajos de clase práctica (laboratorio)

EPE: estudio y preparación de examen final escrito.

RE: realización del examen final escrito.

AT: asistencia a tutorías (reales y/o virtuales). 
Y, como prueba de este compromiso claro de apostar por esta modalidad de aprendizaje, en clara consonancia con las directrices del EEES, la nota final, resultado de una evaluación continua, se calculará a partir de la suma de:

- El control de las actividades propuestas en clase -individuales, cooperativas y de trabajo en equipo (ACT, PTi, PTCo, AT)- supervisadas virtualmente, y evaluadas en clase. El total de esta sección de la nota valdrá como máximo un $50 \%$, distribuyéndose así:

- Participación continua en el blog de la asignatura: $15 \%$;

- Problemas propuestos resueltos: $10 \%$;

- Exposición en clase de tema elaborado en equipo: $25 \%$

- La nota de laboratorio (ACP + PTCP): resolución y entrega de informe y cuestiones (20\%).

- Un examen final escrito o control (PTi + PTCo + EPE + $\mathrm{RE})$, y realizado de forma presencial (30\%). El examen constará de 2 partes: teoría ( $60 \%, 6$ cuestiones) y problemas ( $40 \%, 2$ problemas).

\subsection{Planificación temporal}

Para implementar esta metodología docente en base a un volumen estimado de dedicación del alumno, se diseñó previamente un plan de actividades presenciales y virtuales. Si bien, en algunas ocasiones tuvo que adaptarse a diversas situaciones habituales en un calendario académico, la tabla que se incluye a continuación es la versión definitivamente aplicada en el curso 2007-08.

\section{Resultados: IMPLEMENTACión REAL}

Antes de empezar a describir paso a paso algunos pormenores destacados de la marcha de la asignatura, tanto sus pequeños éxitos como fracasos, conviene remarcar aquí que los alumnos matriculados no fueron advertidos a tiempo en el período de matrícula que esta asignatura iba a impartirse según un paradigma de aprendizaje basado en ellos, como pretende aplicar cualquier metodología docente basada en el aprendizaje semipresencial o b-learning. Esto detalle inicial es muy importante, y clave tanto si los alumnos se matriculan o no conscientemente en asignaturas "pioneras o pilotos" antes de la implantación de las nuevas carreras de grado. Por tanto, la primera clase del curso, centrada en la presentación de la asignatura, se convirtió en la clase más importante de todas. Con tantas novedades para presentar, habia que ir con mucho tiento, dando lo mejor de uno mismo como profesor y líder, para informar y convencer a los alumnos presentes que esta experiencia didáctica que iban a comenzar les beneficiaría en todos los aspectos. Lo que ocurrió en dicha sesión presencial (lunes 11 de febrero de 2008) está bien reflejado en la primera entrada del blog de la asignatura (http:// blogs.ua.es/verduset70/ 2008/02/12/presentacion-de-laasignatura-i/). Aquí se pueden ver información ya descrita (objetivos didácticos, evaluación, etc.) en este artículo, pero también algunos detalles sobre los nuevos roles del alumno y el profesor. A pesar del impacto emocional que supuso esta primera clase (nueva modalidad docente, nuevas herramientas informáticas, exponer en clase a final de curso, etc.), el objetivo de transmitir confianza en la nueva forma de estudiar una asignatura se consolidó a tenor de los hechos posteriores, y que describiré a continuación.

\subsection{Actividades presenciales y virtuales}

Tal como se ha visto en la Tabla 2, el plan de actividades en clase alternaba entre sesiones presenciales y virtuales, en una secuencia clara de sesión presencial + sesión virtual + sesión presencial (de retroalimentación). A este respecto, el hecho de tener las horas de clase presencial siempre los lunes y martes ayudó bastante en la supervisión semanal de todas las actividades individuales y grupales de los alumnos. En la impartición de contenidos teóricos y prácticos (problemas numéricos, etc.) en clase presencial se optó por utilizar la plataforma digital de gestión de contenidos de aprendizaje de la UA (http://www.ua.es/es/univirtual/index.html), conocida habitualmente como Campus Virtual, mediante el uso del plug-in para MSWord denominado CourseGenie (ahora Wimba Create) para la generación del material del curso (temas, cuestiones de retroalimentación, inserción de enlaces web, animaciones, vídeos, etc.). En la Figura 1 se muestra un ejemplo del uso de la herramienta "Sesiones" combinando una ficha de tareas principales (que se repetía siempre en el blog) y el material docente básico. Las tareas principales a supervisar y evaluar por tema no eran nunca más de tres, con dos tipologías básicas: opinión (para el blog) y acción (para el blog, entrega de 
TABLA 2: PLANIFICACIÓN TEMPORAL DE LA ASIGNATURA

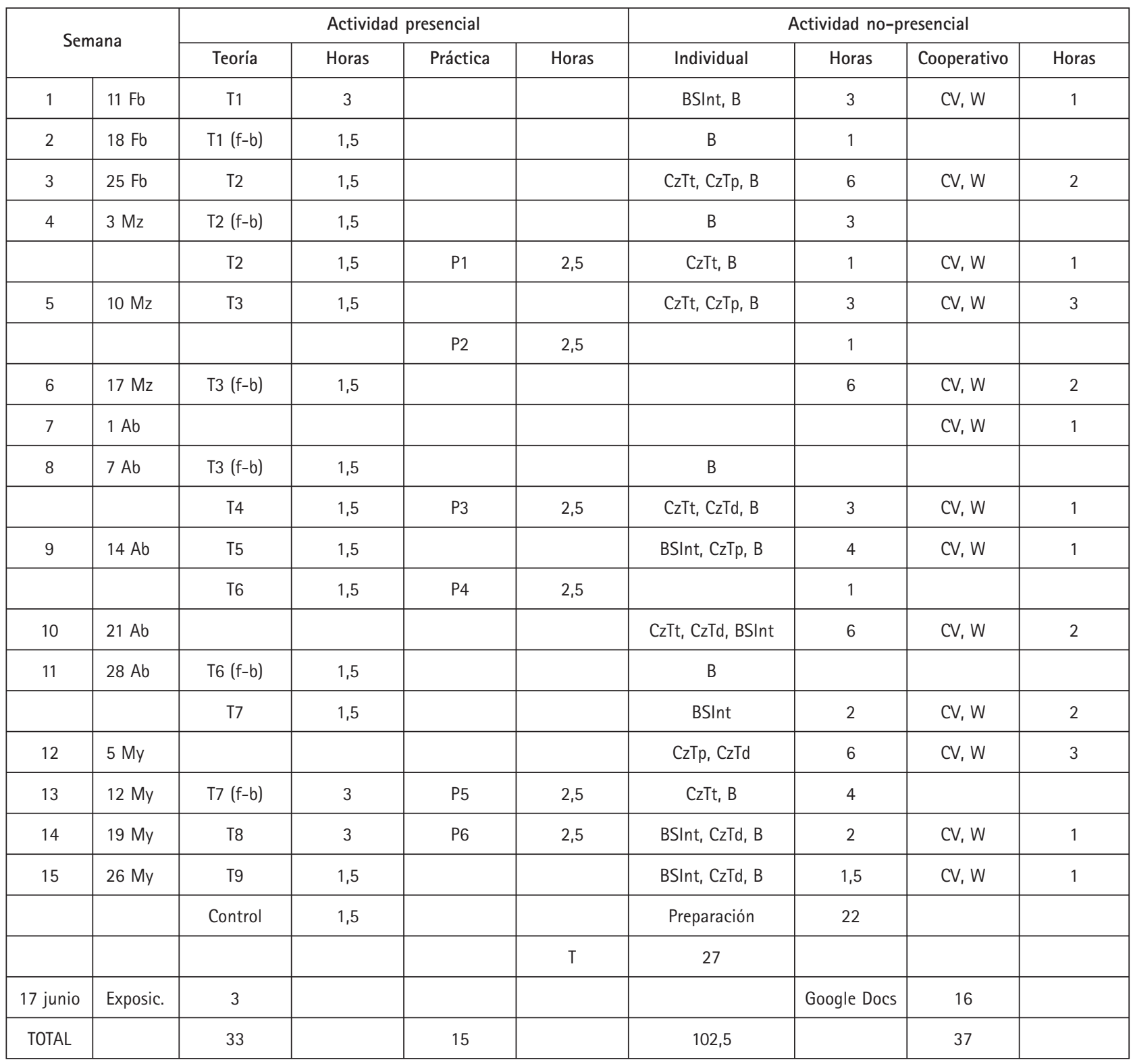

f-b: sesión de puesta en común (feed-back) de las actividades no-presenciales.

BSInt: búsqueda de recursos por Internet.

CzTt: caza del tesoro-test.

CzTp: caza del tesoro-problema (problema numérico).

CzTd: caza del tesoro-debate (CVirtual, blog).

CV: Campus Virtual.

T: tutorías.

W: wiki asociado a la asignatura, en este caso, vía Google Docs.

B: blog asociada a la asignatura. 
problemas, etc.). Así se actuó durante los 4 primeros temas de la asignatura. A partir del tema 5, por motivos de acumulación de tareas en mi actividad docente, investigadora y de gestión, los materiales docentes (temas desarrollados en PowerPoint y convertidos en PDF) de cada tema restante se facilitaron vía el procedimiento habitual que permite el
Campus Virtual para entregar material docente a los alumnos. No obstante, tal como se puede observar en el blog de la asignatura, la ficha de cada sesión de tema se mantuvo hasta el final. Por tanto, para cursos posteriores queda pendiente hacer nuevos materiales docentes en Wimba Create, etc., o reformar los ya existentes.
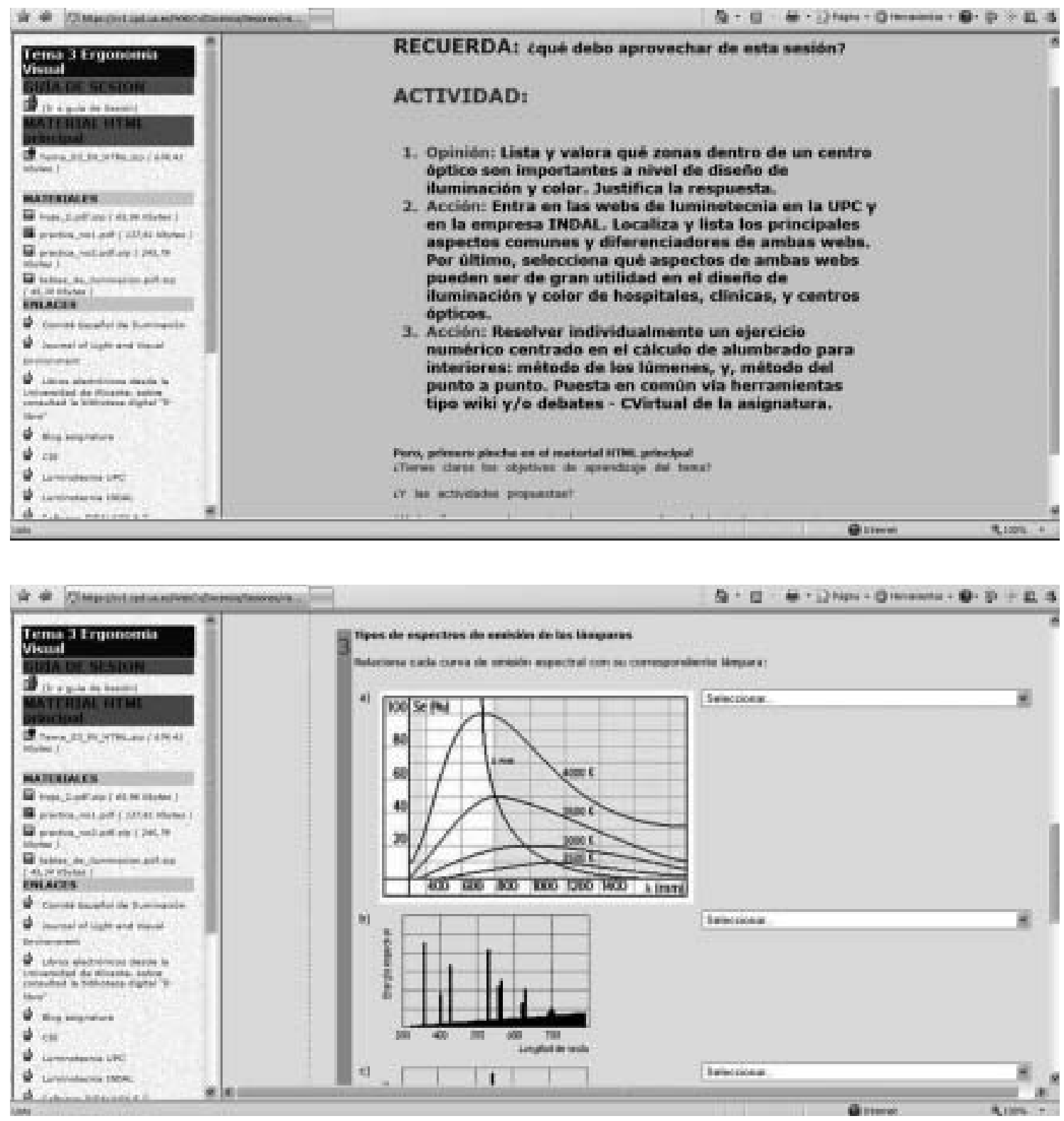

Figura 1. Ejemplos de materiales docentes digitales usados en esta asignatura. Arriba: guía de la sesión para el tema 3 (que se repite en el blog: http://blogs.ua.es/verduset70/2008/03/13/retro-alimentacion-1-tema-3/). Abajo: material hipermedia, en este caso interactivo, utilizado para proporcionar conocimiento teórico y aplicado del tema 3. 


\subsection{Actividades de trabajo en equipo}

Otro aspecto destacable del devenir del curso fue la supervisión de los grupos de trabajo para realizar las exposiciones de varios temas complementarios de la asignatura para final de curso. Recordemos que esta tarea se evaluó con un $25 \%$ del total de la nota. Para ello, como ya sospeché, y dado que era una de las novedades más impactantes de la asignatura, costó bastante articular los equipos y empezar a arrancar con esta tarea. Opté por usar la herramienta GoogleDocs, y tuvo un éxito rotundo, a pesar de que se empezó a desarrollar a mitad de curso. Para tal fin, las instrucciones para planificar el tiempo de dedicación al trabajo cooperativo vía Google Docs del tema a exponer se colgaron en el blog de aula (http://blogs. ua.es/verduset70/2008/ 04/21/trabajo-en-equipo-usandogoogledocs-para-preparar-la-exposicion-en-clase/, Figura 2). Esta tarea también puede hacerse, como he comprobado, dentro de la sección "Debates" del Campus Virtual, abriendo un debate para cada tema de exposición. Pero los alumnos de esta asignatura no tuvieron ningún problema en seguir la herramienta GoogleDocs. En mi papel como profesor, asumí un rol de un miembro más en cada equipo, pero obviamente más experimentado, para supervisar los avances de cada trabajo. De esta forma, ponía en práctica una adaptación propia del liderazgo situacional a la clase, tal como expuse en la entrada "Liderazgo situacional aplicado al aula y aprendizaje informal" (http:// blogs.ua.es/verduset70/2008/04/23/liderazgo-situacionalaplicado-al-aula-y-aprendizaje-informal/). La experiencia como tal fue completamente satisfactoria y enriquecedora tanto para los alumnos como para mí, como expondré con datos objetivos más adelante. Se supervisaron tres grupos/ exposiciones. Al principio, tal como se marcan en las instrucciones anteriores, se proporcionó material informativo de partida (enlaces web, documentos, etc.), incluso algún ejemplo de exposición de cursos anteriores, facilitando así la espiral de gestión de conocimiento en la docencia (http://innovacioneducativa.wordpress.com/2008/12/10/ espirales-de-conocimiento-como-mejorar-el-trabajo-enequipo-y-la-competitividad/). Al final dos de los trabajos se visualizaron vía PowerPoint, y el otro directamente vía GoogleDocs. En la evaluación de estos trabajos se contempló el nivel de contenidos, la relación con la asignatura, la participación individual, el diseño de la presentación, así como el seguimiento continuo. Un aspecto final muy

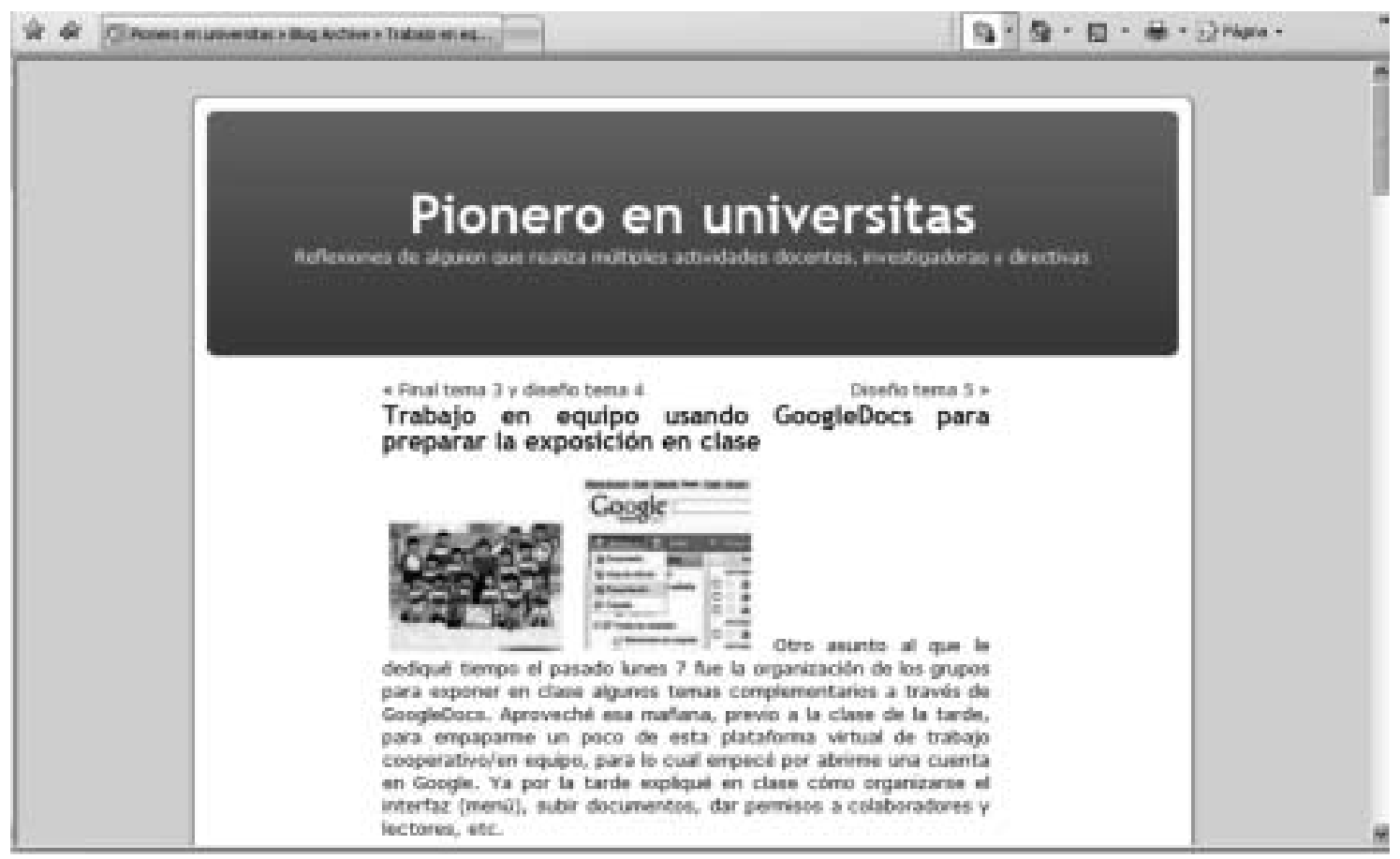

Figura 2. Instrucciones para realizar en equipo exposiciones en clase a final de curso. 
importante sobre esta actividad fue que tales exposiciones se realizaron en la fecha oficial del examen (mediados de junio), con una duración total de 3 h., que permitió que los grupos expusieran con tranquilidad (no más de 15 min.), pero que también diera tiempo para los debates en cada tema de exposición (que yo utilicé también como última sesión presencial de retroalimentación). Por tanto, tal como se indica al final en la Tabla 2, la última clase presencial se dedicó a realizar el control (examen) final escrito.

Dentro de esta misma sección, se pueden incluir las sesiones presenciales y la dedicación posterior de las prácticas de laboratorio, con la replicación de montajes experimentales y material informático para que todos los grupos realizaran la misma actividad programada. Esto corresponde a una actividad docente convencional, y en la evaluación de las mismas ( $20 \%$ del total de la nota) solamente se contempló la recogida y supervisión de un informe final de una de las seis sesiones de laboratorio, distribuidas aleatoriamente entre cada alumno. A la vista de los objetivos didácticos descritos anteriormente, la finalidad principal de estas sesiones de laboratorio era proporcionar una panorámica general de las reglas de fotometría, el diseño de iluminación de interiores y exteriores, y los ejercicios posibles a desarrollar en entrenamiento visual.

\subsection{Fin de curso y notas}

Como se puede intuir a tenor de lo descrito previamente, la actividad a final de curso para los alumnos era frenética, pero supongo que no agobiante, al mezclarse las actividades de esta asignatura con otras de asignaturas convencionales (anuales y cuatrimestrales). En el último mes de curso, los alumnos ya habían adelantado, unos más otros menos, la realización del trabajo de exposición, y casi todos ellos llevaban al día (realmente a la semana) la realización de las actividades propuestas en cada tema (sobre todo, participar en el blog de aula). De esta forma, a mediados de mayo les avancé ejemplos de exámenes resueltos de cursos anteriores para que fueran asimilando que la última clase presencial sería el "tradicional" examen final (con sólo un $30 \%$ de la nota final). Con esta ayuda extra, creo que aminoré el estrés que podría acumularse en mis alumnos a estas alturas de curso. Como este control escrito no era excluyente, sino una prueba acumulativa más de evaluación (con formulario seleccionado ya incluido), algunos estudiantes se lo prepararon menos, y otros más. La tabla 3 muestra las notas obtenidas:

Respecto a la evaluación continua, resultante de la supervisión de las actividades individuales (participación en blog, problemas, etc.) y grupales (exposiciones), las tablas 4 y 5 , en la página siguiente, muestran los resultados obtenidos:

TABLA 3: RESULTADOS DE LA EVALUACIÓN DEL CONTROL FINAL ESCRITO

\begin{tabular}{|c|c|c|c|c|c|}
\hline N. ${ }^{\circ}$ ALUMNO & TEORÍA /10 & TEORÍA /6 & PROBL. /10 & PROBL. /6 & EXAMEN \\
\hline 1 & 8,33 & 5,00 & 4,50 & 1,80 & 6,80 \\
\hline 2 & 7,92 & 4,75 & 0,00 & 0,00 & 4,75 \\
\hline 3 & & & & & NP \\
\hline 4 & & & & & NP \\
\hline 5 & & & & & NP \\
\hline 6 & 8,33 & 5,00 & 0,00 & 0,00 & 5,00 \\
\hline 7 & 8,33 & 5,00 & 10,00 & 4,00 & 9,00 \\
\hline 8 & 9,58 & 5,75 & 8,75 & 3,50 & 9,25 \\
\hline 9 & 8,75 & 5,25 & 4,50 & 1,80 & 7,05 \\
\hline 10 & 9,17 & 5,50 & 4,50 & 1,80 & 7,30 \\
\hline 11 & 7,50 & 4,50 & 8,75 & 3,50 & 8,00 \\
\hline 12 & 7,08 & 4,25 & 4,00 & 1,6 & 5,85 \\
\hline 13 & & & & & NP \\
\hline
\end{tabular}


TABLA 4: RESULTADOS DE LA EVALUACIÓN CONTINUA

\begin{tabular}{|c|c|c|c|c|c|}
\hline N. ${ }^{\circ}$ ALUMNO & OPINION & PROBL. & ACTIV. & EXPOS. & TOTAL /10 \\
\hline 1 & 9 & 10,00 & 7,78 & 8,50 & 5,71 \\
\hline 2 & 3 & 0,00 & 1,11 & 9,00 & 0,02 \\
\hline 3 & 0 & 0,00 & 0,00 & 0,00 & 0,74 \\
\hline 4 & 3 & 0,00 & 2,22 & 0,00 & 0,42 \\
\hline 5 & 2 & 0,00 & 1,11 & 0,00 & 5,27 \\
\hline 6 & 3 & 0,00 & 1,11 & 9,50 & 8,88 \\
\hline 7 & 8 & 10,00 & 6,67 & 9,50 & 8,04 \\
\hline 8 & 9 & 10,00 & 4,44 & 8,50 & 8,81 \\
\hline 9 & 10 & 10,00 & 7,78 & 8,50 & 7,84 \\
\hline 10 & 7 & 10,00 & 4,44 & 8,50 & 8,48 \\
\hline 11 & 9 & 10,00 & 6,67 & & 6,95 \\
\hline 12 & 7 & 6,67 & 3,33 & 1,11 & \\
\hline 13 & 3 & 0,00 & & 0,50 & \\
\hline
\end{tabular}

TABLA 5: RESULTADOS DE LA EVALUACIÓN FINAL

\begin{tabular}{|c|c|c|c|c|}
\hline N. ${ }^{\circ}$ ALUMNO & EXAMEN & PRÁCTICAS & EVAL. CONT. & TOTAL / 10 \\
\hline 1 & 6,80 & 8,00 & 8,71 & 8,0 \\
\hline 2 & 4,75 & 5,00 & 5,02 & 5,0 \\
\hline 3 & NP & & 0,00 & NP \\
\hline 4 & NP & 0,74 & NP \\
\hline 5 & NP & & 0,42 & NP \\
\hline 6 & 5,00 & 5,27 & 5,7 \\
\hline 7 & 9,00 & 8,00 & 8,88 & 9,1 \\
\hline 8 & 9,25 & 10,00 & 8,04 & 8,4 \\
\hline 9 & 7,05 & 8,00 & 8,81 & 8,1 \\
\hline 10 & 7,30 & 8,00 & 7,84 & 7,7 \\
\hline 11 & 8,00 & 8,00 & 8,48 & 8,2 \\
\hline 12 & 5,85 & 8,00 & 6,95 & 6,4 \\
\hline 13 & NP & 6,00 & 0,52 & NP \\
\hline
\end{tabular}

Como se puede ver en estas tablas, algunas conclusiones pueden extraerse:

- 9 de 13 alumnos matriculados participaron activamente en la asignatura. Dos de estos nueve alumnos, los codificados como "2" y "6", tuvieron una evaluación continua pequeña, pero más que suficiente para aprobar la asignatura compensando con otras secciones de evaluación.

- La participación en el blog de aula en el formato "opinión" fue muy alta. De 17 entradas del profesor y 9 temas explicados, el promedio de intervenciones coherentes de los alumnos fue de 6 . 
- La participación en el blog de aula en el formato "acción" (actividad) también fue muy alta, con un nota promedio de 4.81 (descartando a los alumnos NP) para 9 actividades propuestas.

- Más de la mitad de los alumnos entregaron más de dos problemas numéricos propuestos (de un total de 3).

- Las notas del control escrito fueron muy positivas para los alumnos presentados.

- Las notas de exposición fueron altas y merecidas, siendo para todos la primera vez que hablaban en público encima del "púlpito del profesor", pero también gracias a la labor gratificante de supervisión que realicé en cada equipo.

- En definitiva, que todos aquellos que participaron y fueron evaluados (9 de 13) en cada una de las actividades principales propuestas (control escrito, prácticas y evaluación continua) aprobaron la asignatura, o sea, un porcentaje real de aprobados del 100\%.

Respecto a la recuperación de la asignatura, aspecto que tampoco debemos olvidar y que tenemos que prever, aunque sea una metodología b-learning, se avisó desde el principio que todo alumno debía puntuar en cada actividad principal. Cada sub-nota principal no era excluyente, sino acumulativa, a no ser que no hubiera registro. Esto quiere decir que si un alumno acumula nota suficiente a 5 ptos. con el control escrito y las prácticas, pero no ha participado en la exposición de final de curso, no se le aprobará, sino que se codifica como NP, y se le guarda la nota acumulada para la convocatoria de recuperación (septiembre). Pues bien, ninguno de los cuatro estudiantes NP se apuntaron a la convocatoria de septiembre. Cabe decir, para finalizar esta puntualización, que también está relacionada con las bajas producidas en la asignatura, que justamente estos cuatro estudiantes, que casi nunca asistieron a clase, expresaron una indiferencia absoluta desde principios de curso al conocer las innovaciones educativas que incorporaba, y suponer por ello un plan de trabajo continuo a lo largo del cuatrimestre, para el cual no estaban prestos a hacer sin cambiar sus prioridades previstas.

\section{Valoraciones finales}

Un profesional docente competente no puede dejar de ser reflexivo sobre su actividad. $Y$ a este respecto no dejé desaprovechar la oportunidad del día de las exposiciones (mediados de junio), una vez que todos los alumnos habituales ya podian valorar coherentemente toda la asignatura, para recoger sus opiniones sobre el periodo/proceso docente compartido con ellos. A este respecto, preparé una encuesta propia, que adjunto como Anexo, con los siguientes aspectos a evaluar:

- esfuerzo y tiempo dedicado por los alumnos en la asignatura, tanto de la parte teórica como de la práctica, y tanto de la parte presencial como la de la no presencial.

- puntos fuertes y débiles,

- y sugerencias de mejora.

Por tanto, aproveché las nueve encuestas informales de opinión para autorreflexionar sobre mi labor docente y tomar, si cabe, algunas decisiones de cambio, siempre para mejorar lo ya establecido. Del análisis de los resultados de las encuestas cabe resaltar:

- Sobre contenidos/documentación: notas muy positivas, la asignatura es interesante y útil, tal como ya refleja las directrices ministeriales para el futuro Grado en Óptica y Optometría.

- Sobre metodología: se ha asimilado bien, pero ha costado puesto que no estaban preparados para ello. Aun así, el formato de evaluación ha sido altamente reconocido.

- Profesorado y coordinación académica: los alumnos se sienten altamente satisfechos con el asesoramiento y la orientación que ha realizado el profesor en base a sus requerimientos individuales.

- Estudiante: a pesar del esfuerzo y el tiempo dedicado a una asignatura optativa (que infravaloraban inicialmente), los grados de implicación y satisfacción de lo vivido y aprendido en la asignatura han sido altos.

- Respecto a la pregunta 5 ("¿recomendarías esta asignatura a otras personas?): sí a nivel de contenidos y relación con la carrera universitaria, siempre y cuando se informe previamente al alumnado sobre la nueva metodología docente empleada. Por tanto, si el estudiante no está preparado o habituado ya en la nueva metodología b-learning que promulgan las directrices del EEES, es muy probable que no se decante por seleccionar esta asignatura optativa. Y así ha ocurrido en la matrícula del curso 2008-09, donde nadie se ha matriculado. 
- Puntos fuertes: se aprende mucho, al ritmo que desee el estudiante, y las nuevas herramientas digitales (CVirtual, blog, etc.) ayudan a interactuar y aprender mejor.

- Puntos débiles: demasiadas actividades, se debe estimar mejor el tiempo de dedicación del alumno.

- Sugerencias de mejora: limitar las actividades no presenciales.

Respecto a mi valoración personal:

- Obviamente, no se puede siempre superar un reto al primer intento y sentirse satisfecho del todo, pero, en mi caso, debo confesar que la experiencia ha sido muy gratificante. Así, por ejemplo, el rendimiento académico de este curso comparado con los anteriores ha sido incluso ligeramente superior.

- A pesar del tiempo invertido, sobre todo en la preparación de nuevos materiales docentes, cuando se ya se disponía de ellos en otros formatos (PowerPoint, etc), estoy convencido de haber asentado bien esta metodología docente en esta asignatura, y, saber implementarla en otras. Con lo cual, en próximas ediciones de la misma se potenciarán sus puntos fuertes y se minimizarán sus puntos débiles, sobre todo limitando bien las actividades no presenciales (por ejemplo de tres a dos por tema, y, trabajando con "debates" del CVirtual para realizar el seguimiento de las exposiciones, y no vía GoogleDocs).

- Sin embargo, estoy bastante desanimado con la falta de continuidad en esta asignatura al ser de carácter optativo, y por ejemplo no tener alumnos en el curso actual (200809). Es verdad que como asignatura optativa es muy amplia en contenidos debido a su impronta inter y multidisciplinar. Lo ideal sería dividirla en varias asignaturas más, al menos en dos separando radiometría/fotometría (temas actuales 1-5) y rendimiento visual (temas actuales 6-10).

- Para colmo, aun siendo una materia recomendada en las directrices ministeriales de esta carrera universitaria, incluida en el bloque conceptual de Optometría, dudo mucho que en la actual comisión de grado, que actúa en la Universidad de Alicante para el diseño del plan de estudios del futuro Grado en Óptica y Optometría, la mantenga como está, sino que seguramente será disgregada en temas sueltos dentro de otras asignaturas troncales y optativas.

- Por tanto, da para pensar que se ha perdido en parte el tiempo, si bien estoy convencido que este modo de actuar como profesional docente, y con esta nueva metodología y saber actuar, es el correcto y seguiré haciéndolo en asignaturas presentes y futuras, asesorando sin inconvenientes a colegas que estén dispuestos a escuchar y valorar (no necesariamente seguir) mis experiencias y consejos.

- Espero, pues, que los aspectos positivos de esta experiencia educativa se puedan extrapolar con mayor éxito en otros estudios universitarios y preuniversitarios. En cualquier caso, siempre estaré a la disposición de aquellas personas inquietas por la innovación educativa, ya sea pre o universitaria.

- A la vista de mi experiencia educativa, y teniendo en cuenta la zona de rendimiento óptimo adaptada al aprendizaje que enuncia D. Goleman en su reciente monografía "Inteligencia social" (Ed. Kairós, 2006, página 351), estoy convencido de que las nuevas TICs de apoyo a la docencia pueden aportar muchos recursos para adaptar los retos de aprendizaje a un nivel proporcional al alcance de las capacidades individuales de cada estudiante. Este trabajo es un claro ejemplo de implementación práctica, con mayor o menor éxito, según como se mire. Sin embargo, esta misma implementación puede que no sea tan fácil para grupos más numerosos de estudiantes y disciplinas pre y universitarias. Éste será el gran reto para los próximos años si no se optimizan eficientemente los recursos instrumentales y humanos, ya disponibles y otros necesarios, en la educación universitaria. 


\section{AGRADECIMIENTOS}

A la Universidad de Alicante, a su Vicerrectorado de Tecnología e Innovación Educativa y al Instituto de Ciencias de la Educación, por su respaldo institucional, formación complementaria (cursos de blearning, liderazgo situacional, guías docentes, etc.) y asesoramiento técnico en innovación educativa. Al profesor Ángel Fidalgo por su estímulo y asesoramiento.

\section{REFERENCIAS}

Boyce, P. R. (2003): Human factors in lighting, 2nd ed., London: Taylor \& Francis.

Comisión para la renovación de las metodologías docentes en la Universidad (CCU-MEC) (2006): Propuestas para la renovación de las metodologías docentes en la Universidad, http:// www.mec.es/educa/jsp/plantilla. jsp?area $=$ ccunivetid $=910$.

Fidalgo, A. (2008): Blog de Innovación educativa, http://innovacioneducativa. wordpress.com/.

Lillo Jover, J. (2000): Ergonomía. Evaluación y diseño del entorno visual, Madrid: Alianza Editorial, Psicología y Educación.

Martínez-Verdú, F. M.: Blog Pionero en universitas, http://blogs.ua.es/verduset70/category/ergonomia-visual/

Michavila, F. (2004): Estudio sobre innovaciones educativas basadas en las tecnologías de la información en la formación universitaria presencial y a distancia, http://liti.dmami.upm.es/ elearning2004/.

North, R.V. (2001): Work and the Eye, 2nd ed., Oxford: Butterworth-Heinemann.

Smith, N. A. (2000): Lighting for Health and Safety. Oxford: Butterworth-Heinemann.
Recibido: 9 de marzo de 2009 Aceptado: 16 de abril de 2009 


\section{ANEXO: Ficha de encuesta informal de opinión sobre el proceso docente}

\section{ENCUESTA FINAL}

Para conocer vuestra opinión relacionada con la acción formativa en la cual habéis participado, y poder ayudarnos a mejorar futuras acciones, os agradeceriamos que rellenáseis este cuestionario que os proponemos a continuación.

Señalar con una "X" aquel valor con el que os sentís más identificad@s:

$$
(1=\text { Poco } \quad / \quad 2 \text { = Suficiente } / \quad 3 \text { = Bastante } / \quad 4 \text { = Notable } \quad / \quad 5 \text { = Mucho })
$$

\section{Asignatura: ERGONOMÍA VISUAL}

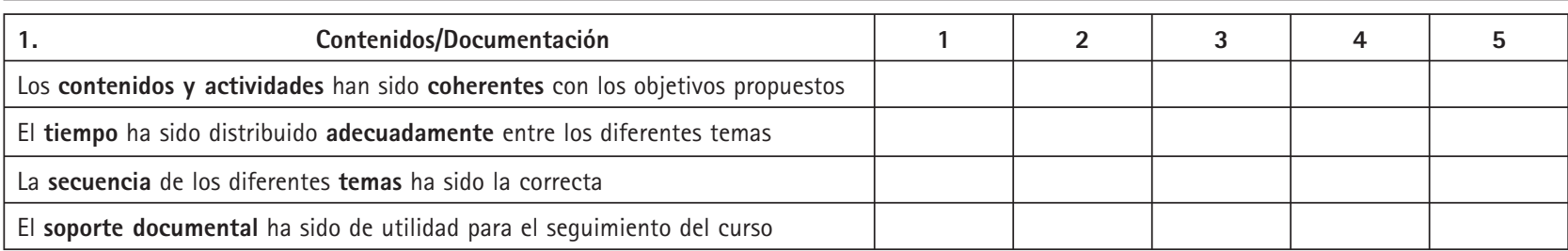

\begin{tabular}{|c|c|c|c|c|c|}
\hline 2. Metodología & 1 & 2 & 3 & 4 & 5 \\
\hline \multicolumn{6}{|l|}{ Ha existido un equilibrio coordinado entre los contenidos teóricos y prácticos } \\
\hline \multicolumn{6}{|l|}{ Se ha estimulado el interés por los diferentes temas } \\
\hline \multicolumn{6}{|l|}{ Se ha fomentado la participación/comunicación entre los asistentes } \\
\hline \multicolumn{6}{|l|}{ La metodología utilizada ha facilitado la asimilación de los contenidos } \\
\hline $\begin{array}{l}\text { El formato de evaluación de los aprendizajes es coherente y adecuado para la } \\
\text { metodología docente utilizada }\end{array}$ & & & & & \\
\hline
\end{tabular}

\begin{tabular}{|c|c|c|c|c|c|}
\hline 3. Profesorado y coordinación académica & 1 & 2 & 3 & 4 & 5 \\
\hline El conocimiento de la materia del profesorado ha sido adecuado al curso & & & & & \\
\hline El asesoramiento y orientación necesarios para el alumno han sido satisfechos & & & & & \\
\hline La coordinación entre los profesores y coordinadores ha sido satisfactoria & & & & & \\
\hline
\end{tabular}

\begin{tabular}{|c|c|c|c|c|c|}
\hline 4. Estudiante & 1 & 2 & 3 & 4 & 5 \\
\hline \multicolumn{6}{|l|}{ El tiempo necesario de dedicación para lograr aprobar la asignatura es: } \\
\hline \multicolumn{6}{|l|}{ El grado de esfuerzo invertido por ti para aprobar la asignatura ha sido: } \\
\hline \multicolumn{6}{|l|}{ El uso de recursos informáticos para seguir el curso ha sido acertado } \\
\hline \multicolumn{6}{|c|}{ Tu grado de participación/implicación ha sido más de lo esperado al principio } \\
\hline Tu grado de satisfacción sobre lo vivido y aprendido en el curso es & & & & & \\
\hline
\end{tabular}

\section{5. ¿Recomendarías esta asignatura a otras personas? ¿Por qué?}

\section{Otras observaciones sobre los puntos positivos y negativos de este curso.}

\section{Sugerencias de mejora para la asignatura.}

